\title{
Modeling Propagation of COVID-19 in the UK
}

\author{
Babak Jamshidi, PhD; Hakim Bekrizadeh, PhD; Shahriar Jamshidi Zargaran, MSc; \\ Mansour Rezaei, PhD
}

Key Words: COVID-19, modeling, propagation

$\mathrm{C}$ oronavirus disease (COVID-19) was first identified in December 2019 in Wuhan, China. From the beginning, this disease has been the subject of various scientific studies. Due to the large amount of data related to the spread of COVID-19 and the high speed of changes, particularly in modeling and forecasting works, it is required to update the predictions and assess the goodness of performance or the accuracy of the models. In this regard, we aim at evaluating the performance of the model introduced by Jamshidi et al. ${ }^{1}$ to describe the first wave of infectious diseases. Since about the propagation of COVID-19 in the UK, until early July 2020, we had encountered the first wave of the disease, and it is possible to examine the performance of the model to describe the trend of the disease up to early July. Therefore, in this letter, we want to evaluate the performance of the model in 2 periods:

- The time studied by Jamshidi et al. ${ }^{2}$ (April 15 to May 30, 2020)

- A 1-month period thereafter (May 31 to July 1)

\section{PREDICTION VERSUS REALITY}

Based on the calculations of Jamshidi et al., ${ }^{2}$ the following estimated parameters for the model were obtained to describe the daily relative increment of confirmed cases and case fatality rate, respectively:

$$
\begin{gathered}
(\widehat{b}, \widehat{I R}, \widehat{K}, \widehat{\theta}, \widehat{a})=(13,0.3272,88.2050,1.9283,0.3382) \\
(\widehat{b}, \widehat{I R}, \widehat{K}, \widehat{\theta}, \widehat{a})=(0,0,0.5430,0.2427,0.0869)
\end{gathered}
$$

The above estimations were based on the time series of the number of confirmed cases and deaths until April 14, 2020. Jamshidi et $\mathrm{al}^{2}{ }^{2}$ applied the above models to forecast the propagation of the pandemic in the UK from April 15 to May 30, 2020. Accordingly, they yielded $282 \mathrm{~K}$ and $31 \mathrm{~K}$ as point estimations and $242-316 \mathrm{~K}$ and $28-50 \mathrm{~K}$ as $80 \%$ confidence intervals for the cumulative number of confirmed cases and deaths on May 30, 2020, respectively. By repeating the simulation, we obtained $273 \mathrm{~K}$ and $243-300 \mathrm{~K}$ (Figure 1A) and $36 \mathrm{~K}$ and $32-43 \mathrm{~K}$ (Figure $1 \mathrm{~B}$ ) as predictions. Since, there were 250347 cases and 37529 deaths reported in the UK by the mentioned date, and in reality, both of the intervals include the real data, and the relative error of the 2 point estimations are $12 \%$ and $9 \%$ and $16 \%$ and $5 \%$ for cases and deaths, respectively.

Similarly, by considering the data up to May 30 and calculating the model fit for them, we get the following estimated parameters to describe the daily relative increment of confirmed cases and case fatality rate, respectively:

$$
\begin{gathered}
(\widehat{b}, \widehat{I R}, \widehat{K}, \widehat{\theta}, \widehat{a})=(13,0.3272,5514.7,2.9317,0.0630) \\
(\widehat{b}, \widehat{I R}, \widehat{K}, \widehat{\theta}, \widehat{a})=(0,0,0.3678,0.1568,0.0268)
\end{gathered}
$$

According to these models, we obtained $291 \mathrm{~K}$ and $279-304 \mathrm{~K}(90 \%$ confidence interval) as forecasts of the cumulative number of reported cases in the UK on July 1, 2020, which was 284000 . Similarly, the point estimation of $45 \mathrm{~K}$ and the $90 \%$ confidence interval of $38-53 \mathrm{~K}$ were obtained as the forecast of the cumulative number of deaths in the UK on July 1, which was 40490. Accordingly, both of the intervals include the real data, and the relative errors of the point estimations are less than $3 \%$ and $11 \%$, respectively.

\section{About the Authors}

Department of Biostatistics, Kermanshah University of Medical Sciences, Kermanshah, Iran (Dr Jamshidi); Department of Statistics, Payam-eNoor University, Iran (Dr Bekrizadeh); Department of Medical Engineering, Tehran University of Medical Sciences, Tehran, Iran (Mr Jamshidi Zargaran) and Social Development and Health Promotion Research Center, Kermanshah University of Medical Sciences, Kermanshah, Iran (Dr Rezaei).

Correspondence and reprint requests to Mansour Rezaei, Social Development and Health Promotion Research Center, Kermanshah University of Medical Sciences, Kermanshah, 6719851351, Iran (e-mail: mrezaei@kums.ac.ir). 
Performance of the Point Estimation and $80 \%$ Confidence Interval Based on the Obtained Model in Forecasting the Cumulative Number of Confirmed Cases and Deaths in the UK on May 30, 2020.

(a)

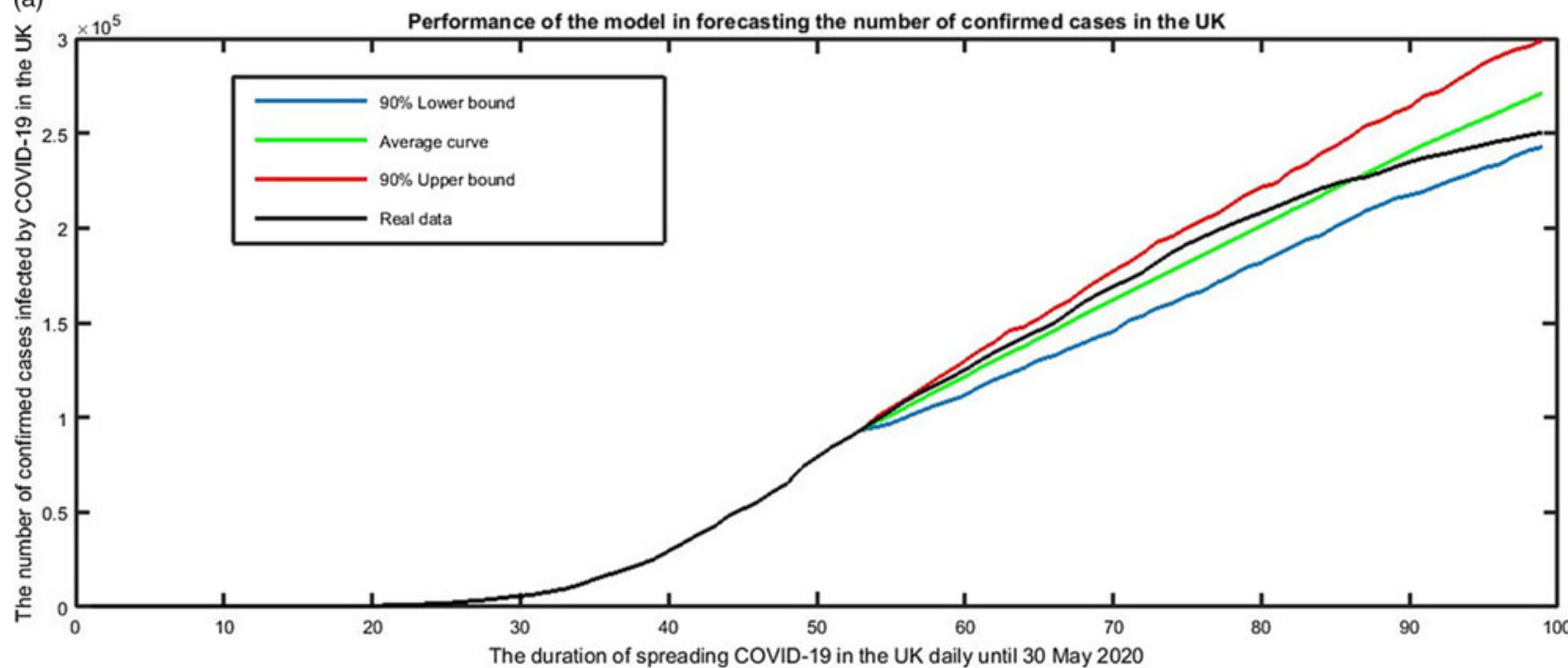

(b)

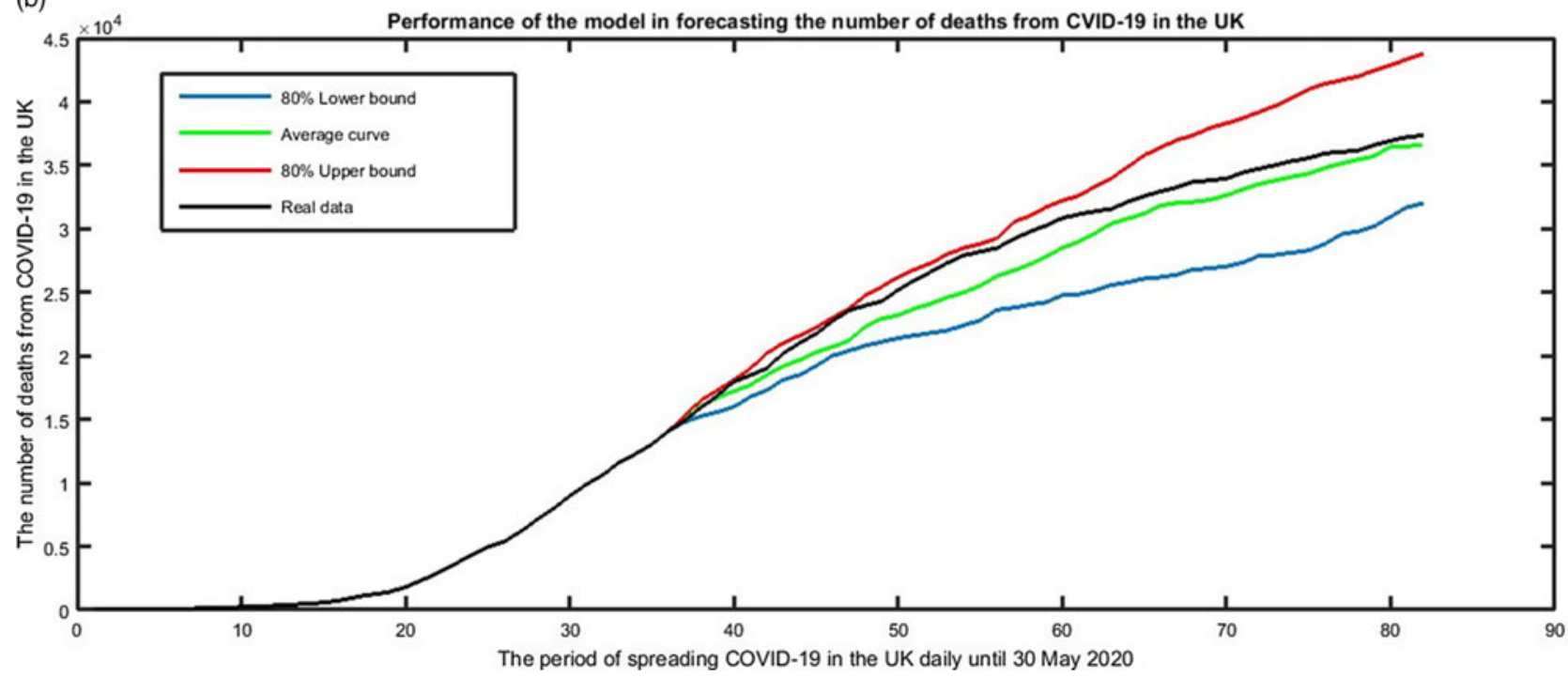

\section{Conflict of Interest Statement}

The authors have no conflicts of interest to declare.

\section{REFERENCES}

1. Jamshidi B, Rezaei M, Bekrizadeh H, Jamshidi Zargaran S. A new family of time series to model the decreasing relative increment of spreading of an outbreak: focused on COVID-19 in China. ResearchGate. 2020. https:// www.researchgate.net/publication/340236985_Modeling_decrease_rate_ of_increment_by_time_series.

2. Jamshidi B, Rezaei M, Kakavandi M, Jamshidi Zargaran S. Modelling the number of confirmed cases and deaths from COVID-19 pandemic in the UK and forecasting over April 15th-May 30th, 2020. Disaster Med Public Health Prep. 2020;epub, 1-17. doi: 10.1017/dmp.2020.312. 